\title{
Model-Based Phase Velocity and Attenuation Estimation in Wideband Ultrasonic Measurement Systems
}

\author{
Jesper Martinsson, Johan E. Carlson, and Jan Niemi
}

\begin{abstract}
A parametric method to estimate frequencydependent phase velocity and attenuation is presented in this paper. The parametric method is compared with standard nonparametric Fourier analysis techniques using numerical simulations as well as real pulse-echo experiments. Approximate standard deviations are derived for both methods and validated with numerical simulations. Compared to standard Fourier analysis, the parametric model gives considerably lower variance when estimating attenuation and phase velocity. In contrast to nonparametric techniques, the proposed estimator avoids the phase unwrapping problem because analytical expressions for the continuous phase velocity and attenuation can be derived.
\end{abstract}

\section{INTRODUCTION}

$\mathrm{I}_{\mathrm{N}}^{\mathrm{N}}$ $\mathrm{N}$ ultrasonic measurement systems, estimates of the frequency-dependent attenuation and phase velocity are often used to deduce the properties of interest. Accurate estimates of these quantities, therefore, are important. However, estimating attenuation and phase velocity from noisy measurements, using standard nonparametric techniques, often results in biased estimates with high uncertainties. The bias occurs when the estimator (a nonlinear function) is applied on the measured data, skewing the shape of the probability density function (PDF) of the measurement noise. The high uncertainties and the erratic behavior of the estimates are drawbacks of using nonparametric methods, e.g., the discrete Fourier transform (DFT), to estimate spectral densities [1]. For nonparametric methods, the number of parameters are equal (or proportional) to the signal sample size; hence, no averaging is done to reduce the variance of the estimated quantities. As a consequence, the variance of the estimate will not tend to zero, as the signal sample size grows, as in parametric methods, but to a constant level that is inversely proportional to the signal-to-noise ratio (SNR) [2].

Using nonparametric techniques, additional uncertainties are introduced when estimating the phase velocity from a wrapped phase. Although different approaches exist to cope with wrapped phases [3]-[6], they all are based on nonparametric spectral estimates and, hence, suffer from bias and high uncertainties in low SNR frequency regions. In [7], the authors investigate errors generated by nonlin-

Manuscript received March 30, 2006; accepted August 9, 2006.

The authors are with EISLAB, Department of Computer Science and Electrical Engineering, Luleå University of Technology, SE-971 87 Luleå, Sweden (e-mail: Jesper.Martinsson@csee.ltu.se).

Digital Object Identifier 10.1109/TUFFC.2007.219 earities when estimating phase velocity and attenuation, including also the timing jitter in a through-transmission setup.

In this paper, a parametric phase velocity and attenuation estimator is proposed. The method is built on a parameterization of the linear dispersion system that describes the relationship between two measured ultrasonic signals. The idea is to reduce the variance of this relationship before it is applied to the nonlinear functions (for calculating phase velocity and attenuation), to obtain lower uncertainties and to prevent bias, i.e., not skewing the PDF (statistical linearity). The parameterization is based on the ideas behind a common, finite-order approximation [8] to the solutions of different measurement setups. The result is a common model structure expressed as a continuous, rational, transfer function in which the parameters are estimated using standard system identification techniques. The main advantages of this parameterization are: higher accuracy, lower uncertainties, control over the phase unwrapping ambiguity, and a large data reduction (because the system is completely described by the set of model parameters).

The paper is organized as follows: In Section II, the wave propagation model is described for dispersive media, and its dependence on specific measurement setups and boundary conditions is discussed. The models are generalized into a linear systems notation from which attenuation and phase velocity are deduced. The nonparametric and parametric estimation techniques are described and approximative expressions for the standard deviations for the estimated attenuation and phase velocity are given for both methods. In Section III computer simulations are used to compare the two techniques and to validate the approximative uncertainty expressions. Experimental results are found in Section IV followed by a discussion and some concluding remarks.

\section{THEORY}

\section{A. Physical Model}

The basic model for acoustic waves in a fluid is obtained using the linearized continuity equation, the linearized force equation, and a relationship between the acoustic pressure and condensation. For linearly dynamic fluids, this relationship is described by a linear system, and a lossy wave equation is obtained: 


$$
\frac{\partial^{2} P(x, \omega)}{\partial x^{2}}=-k^{2}(\omega) P(x, \omega)
$$

where $P(x, \omega)$ is the Fourier transform of the acoustic pressure $p(x, t)$ and $k(\omega)$ is the complex frequency dependent wave number. Here the arguments $x, t$, and $\omega$ are spatial, time, and angular frequency variables, respectively. The ordinary differential equation (ODE) in (1) has the solution:

$$
P(x, \omega)=A_{1}(\omega) e^{-j k(\omega) x}+A_{2}(\omega) e^{j k(\omega) x},
$$

where $j=\sqrt{-1} . A_{1}(\omega)$ and $A_{2}(\omega)$ are to be determined by the boundary conditions.

Different measurement setups result in different boundary conditions. For example, in a through-transmission setup, assuming Neumann boundary conditions (perfect reflectivity), the following linear dynamic relationship is obtained between the transmitted and the received signal:

$$
Y(\omega)=\cosh (j k(\omega) d) U(\omega),
$$

where $U(\omega)=P(0, \omega)$ and $Y(\omega)=P(d, \omega)$ are the Fourier transformed acoustic pressures at distance $x=0$ (transmitted signal) and at distance $x=d$ (received signal), respectively.

Using a pulse-echo setup, there usually is only one sensor that works both as a transmitter and receiver. For this setup, an additional criterion of separable echoes is needed to use an input-output (first and second echo) description. For Neumann boundary conditions, the relationship can be written as:

$$
Y(\omega)=e^{-j k(\omega) d} U(\omega),
$$

where $U(\omega)=P(0, \omega)$ and $Y(\omega)=P(d, \omega)$ are the Fourier transformed acoustic pressures of the first and second echo, respectively.

\section{B. Linear System}

The relationships in (3) and (4) can be written in linear input-output relationship as:

$$
Y(\omega)=H(d, \omega) U(\omega),
$$

where $d$ denotes the distance, and $H(d, \omega)$ is the transfer function connecting the signals. This is the result of assuming linear acoustics.

Focusing on the pulse-echo setup, the transfer function can be rewritten in a magnitude and phase representation as:

$$
\begin{aligned}
H(d, \omega) & =e^{-j k(\omega) d}=e^{\Im\{k(\omega)\} d} e^{-j \Re\{k(\omega)\} d} \\
& =|H(d, \omega)| e^{j \arg \{H(d, \omega)\}},
\end{aligned}
$$

where $\Re\{\cdot\}$ and $\Im\{\cdot\}$ represents the real and imaginary parts.

Using (6), the attenuation $\alpha(\omega)$ and the phase velocity $c_{p}(\omega)$ are defined as:

$$
\begin{gathered}
\alpha(\omega)=-\Im\{k(\omega)\}=-\frac{\log |H(d, \omega)|}{d}, \\
c_{p}(\omega)=\omega / \Re\{k(\omega)\}=-\frac{\omega d}{\arg \{H(d, \omega)\}} .
\end{gathered}
$$

From (7) there are two possible ways to estimate attenuation and phase velocity. The first one is based on calculating the phase velocity $c_{p}(\omega)$ solely from estimates of the attenuation $\alpha(\omega)$, using the Kramers-Krönig relationship between the real and imaginary parts of $k^{2}(\omega)$ [4]. This approach is valid under the assumption that the attenuation and phase velocity are slowly varying functions of frequency. The advantage with this approach is that the phase unwrapping problem, arising when calculating the phase velocity from a wrapped phase, is avoided. However, a good estimate of the attenuation is still needed. The phase unwrapping problem is well-known and has been addressed using other approaches [3], [5], [6].

The second approach, investigated in this paper, is to estimate the transfer function $H(d, \omega)$ in (5) and use this estimate together with (7), without any assumptions made on the dispersion.

\section{Nonparametric Techniques}

The nonparametric technique is perhaps the most commonly used approach to estimate attenuation and phase velocity. It is based on the linear relationship between the input and output signal in (5), the discrete DFT, and the definitions in (7).

The transfer function $H(d, \omega)$ is estimated using the ratio of the Fourier transformed output and input signal. Because the measured signals are both truncated and sampled, the estimate is given by the DFT as:

$$
\hat{H}\left(\omega_{l}\right)=\frac{Y_{N}\left(\omega_{l}\right)}{U_{N}\left(\omega_{l}\right)},
$$

for $\omega_{l}=2 \pi l / N$ and $l=0,1, \ldots, N-1$. Here $\hat{\cdot}$ denotes estimate, and $Y_{N}\left(\omega_{l}\right)$ and $U_{N}\left(\omega_{l}\right)$ are the DFTs of the sampled sequences $\{y[n]\}_{1}^{N}$ and $\{u[n]\}_{1}^{N}$.

With the correct choice of sampling frequency and window size, the continuous ultrasonic pulse can be considered in practice both band and time limited, and the effect of leakage and aliasing errors occurring when truncating and sampling it can be neglected. In this case, the DFT coefficients gives a close to perfect representation of the true spectrum of the pulse at the discrete frequencies $\left(Y\left(\omega_{l}\right) \simeq Y_{N}\left(\omega_{l}\right)\right)$. For this to be fulfilled, the sample size $(N)$ of the measurement window should be chosen so that the system response becomes negligible at the end of the measurement (circular and linear convolution is equivalent).

However, due to additive noise in the measurements, the uncertainty of estimating $H(d, \omega)$ in (5) using (8) is high, and the result is a very erratic function of $\omega_{l}$. This behavior is one of the main drawbacks of using the DFT as estimate of the spectral density [1], [2]. If the estimate of $H(d, \omega)$ is uncertain, the attenuation and phase velocity estimates in (7) will be uncertain as well. 


\section{Parametric Techniques}

In general, a parametric method can be descried as a mapping from the measured data to the estimated parameter vector $\hat{\boldsymbol{\theta}}$, where $\hat{\imath}$ denotes estimate. The parameter vector is connected to a specific model structure $\mathcal{M}$, where for a fixed parameter vector $\boldsymbol{\theta}=\boldsymbol{\theta}_{*}, \mathcal{M}\left(\theta_{*}\right)$ represents one model within the structure $\mathcal{M}$. An important step is to select a model structure $\mathcal{M}$ that represents the true system $\mathcal{S}$. One alternative is to choose a model structure based on solutions to the partial differential equation (PDE), as in (3) or (4), and model $k(\omega, \boldsymbol{\theta})$ from physical characteristics of the media, such as viscosity, heat conduction, and relaxation phenomena [9]. The drawback with this approach is that these model structures are strictly dependent on the specific measurement setup, i.e., the investigated media and the assumed boundary conditions.

An alternative approach is to find a common model structure based on the ideas behind the Mittag-Leffler theorem [8], with an expansion of the transfer functions in (3) and (4) as:

$$
\begin{aligned}
H(x, \omega) & =\cosh (j k(\omega) x)=\sum_{r=0}^{\infty} \frac{(j k(\omega) x)^{2 r}}{(2 r) !} \\
& =e^{j k(\omega) x}=\sum_{r=0}^{\infty} \frac{(j k(\omega) x)^{r}}{r !} .
\end{aligned}
$$

For bandlimited signals, the transfer functions can be approximated very well within the signal's bandwidth, using an expansion (or rational transfer function) of finite order. This leads to a common model structure $\mathcal{M}$ of the form:

$$
H(\omega, \boldsymbol{\theta})=e^{-j \omega \tau} \frac{B(\omega, \boldsymbol{\theta})}{A(\omega, \boldsymbol{\theta})}=e^{-j \omega \tau} \frac{\sum_{q=0}^{n_{b}} b_{q}(j \omega)^{q}}{\sum_{q=0}^{n_{a}} a_{q}(j \omega)^{q}},
$$

where $\boldsymbol{\theta}=\left[\begin{array}{llll}b_{0} & \cdots b_{n_{b}} & a_{0} \cdots a_{n_{a}} & \tau\end{array}\right]^{T}$. Here an additional parameter $\tau$ is introduced to account for pure time delays.

The advantage with this structure is its independence of any prior knowledge concerning the specific boundary conditions and physical properties of the media under investigation. With this ability, it is possible to apply the same structure to various experimental situations. A common drawback with this approximation is that the number of model parameters is increased compared to an accurate physical parametric description (if one exists).

To find the appropriate model order $n_{b}$ and $n_{a}$, a model selection and validation step must be introduced. In the selection process, a set of models with different orders are tested and matched to the system $\mathcal{S}$. The matching or estimation is done using the measured data together with a criterion of fit. How consistent the mapping is depends on: the experimental condition (the data), the model structure, and the criterion of fit. Given a set of matched models, the models are validated using different statistical techniques based on the assumptions made about the true system $\mathcal{S}$.
In this paper, the frequency domain sample maximum likelihood method is used as the criterion of fit, see [10] and Appendix A. The motivation for this choice is its wellbehaved statistical properties with the ability to handle noisy input and noisy output problems (error-in-variable) in measurement setups using repeated burst signals (repeated ultrasonic excitations). For the model selection part, the minimum description length (MDL) criterion [10] is used to find the appropriate order and prevent overparameterization. The model is validated using residual analysis.

\section{E. Uncertainty Bounds}

When estimating a parameter, an expression of the uncertainty of the estimate is very important. Often in ultrasonic measurement setups, it is possible to carry out many repeated experiments or excitations under a short period of time under which the medium under investigation can be assumed to be unchanged.

Given a set of $M$ independent noisy but identically excited experiments, from which a parameter can be estimated, there are different ways to estimate the parameter and its uncertainty. The straightforward way is to estimate the parameter for each noisy experiment, resulting in $M$ estimated parameters, and use the mean value as the final estimate and the sample standard deviation as a measure of its uncertainty. However, this approach is not recommended if the estimator is a nonlinear function of the noisy data because the nonlinearity in general destroys the consistency and efficiency of the estimate [11]. When dealing with nonlinearities, a better way is to first reduce the noise variance by averaging techniques (averaging $M$ experiments), then estimate the parameter by applying the nonlinearity. This approach reduces the bias effect introduced by the nonlinear transformation and results in consistent (as $M \rightarrow \infty$ ) estimates. The consistency property comes at the cost of losing the normality, introduced by averaging the $M$ parameters using the first approach. The approximative uncertainty can be estimated from the derivative of the nonlinearity and the estimated uncertainty of the data.

The expressions in (7) for the attenuation and phase velocity are nonlinear functions of the linear system $H$ in (5); and, on top of that, the estimates of $H$ described in Section II-C and Section II-D are nonlinear functions of the measured data. Given the estimated uncertainty of the measurement noise, the approximate uncertainty of the attenuation and phase velocity can be derived using a first order Taylor approximation as:

$$
\begin{aligned}
\sigma_{\alpha(\omega)}^{2} & \approx 2 \frac{\partial \alpha(H(\omega))}{\partial H(\omega)} \sigma_{H(\omega)}^{2}\left[\frac{\partial \alpha(H(\omega))}{\partial H(\omega)}\right]^{*}, \\
\sigma_{c_{p}(\omega)}^{2} & \approx 2 \frac{\partial c_{p}(H(\omega))}{\partial H(\omega)} \sigma_{H(\omega)}^{2}\left[\frac{\partial c_{p}(H(\omega))}{\partial H(\omega)}\right]^{*},
\end{aligned}
$$

where ${ }^{*}$ denotes Hermitian transpose and $\sigma_{H(\omega)}^{2}$ is estimated from (8) or (11) using the estimated standard de- 

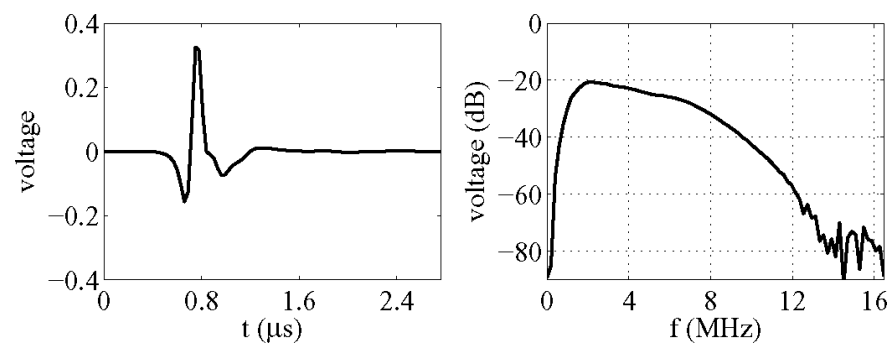

Fig. 1. Mean value of 100 measured first echoes: (left) the sampled time signal; (right) the power spectral density.

viations of the noise and similar approximations as above, see Appendix B. The expressions for the partial derivatives in (12) are found in Appendix B.

\section{Simulations}

In this section the nonparametric and parametric techniques to estimate the attenuation and phase velocity are compared using computer simulations. The validity of the asymptotic expressions in (12) are examined together with the observed numerical standard deviation using 500 independent simulations.

\section{A. The Simulation Model}

To get a similar excitation of the simulated system, compared to the real experiments, the simulated, noise-free input signal (or first echo) was chosen as mean value of 100 measured first echoes from the real experiment, see Fig. 1.

To validate the assumption in Section II-B, that the true model can be approximated with a rational transfer function of finite order, the simulated model structure did not belong to the rational transfer function family. The simulation model was constructed as:

$$
H(\omega, \boldsymbol{\theta}, x)=e^{-j k(\omega, \boldsymbol{\theta}) x}=e^{-j \omega x / c(\omega, \boldsymbol{\theta})},
$$

where $x$ is the distance between the signals and $c(\omega, \boldsymbol{\theta})$ is the complex speed of sound. In the simulation, the complex speed of sound was modeled as [9]:

$$
c(\omega, \boldsymbol{\theta})=\frac{c_{0}}{\sqrt{2}}\left[1+j \omega \eta+\frac{1+j \omega \tau_{1}}{1+j \omega \tau_{2}}\right]^{1 / 2},
$$

with $\boldsymbol{\theta}=\left[\begin{array}{llll}c_{0} & \eta \tau_{1} & \tau_{2}\end{array}\right]^{T}$ as a parameter vector containing the thermodynamic speed of sound $\left(c_{0}\right)$, viscosity $(\eta)$, and two additional parameters $\left(\tau_{1,2}\right)$ contributing to a simulated relaxation phenomenon. In the simulations, the parameters were set to $\boldsymbol{\theta}=\left[1500,1 \cdot 10^{-12}, 3.75 \cdot 10^{-8}, 3.7125\right.$. $\left.10^{-8}\right]^{T}$.

Before the estimation procedure was started, an equal amount of white Gaussian measurement noise was added to the simulated input and output signal. The same SNR, $14 \mathrm{~dB}$ relative to the input signal, was used as estimated in the real experiments. Each simulation consists of 100 repeated noisy excitations, similar to the real experiment.

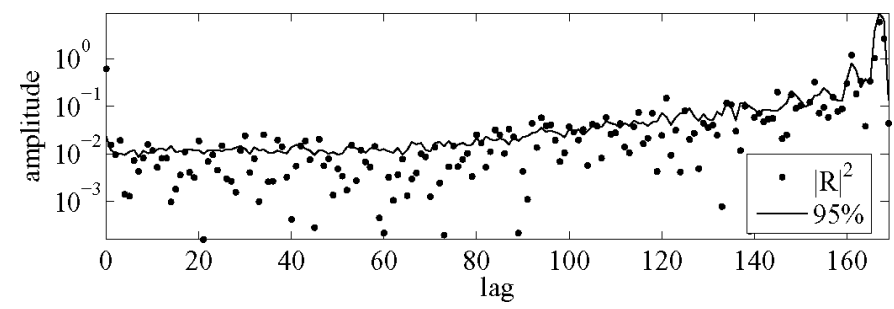

Fig. 2. Whiteness test of the residuals. The residuals are the difference between the modeled and measured transfer functions weighted by its standard deviation [10]. The dots are the amplitude of the cross correlation $R$ of the residuals, and the line marks the $95 \%$ confidence level, where $|R|^{2} \sim \chi^{2}(2)$.

\section{B. Model Selection and Validation}

For the parametric estimation technique, described in Section II-D, a model selection and a validation step were added to find the appropriate model order. The model selection procedure, using the MDL [10], indicates that a model order of 3 , or $n_{a}=n_{b}=3$ is enough to capture the dynamics of the true simulated system within the excited frequency band. The residual analysis in the model validation step can be seen in Fig. 2. The residual is the difference between the modeled (parametric) transfer function and the measured (nonparametric) transfer function weighted by its standard deviation. For a model order of 3 , the whiteness within the $95 \%$ bound might be questionable. However, the final value of the cost function is within its $95 \%$ confidence interval, indicating no model errors. Increasing the model with one order gives higher confidence on the whiteness test, but the final value of the cost function exceeds its lower $95 \%$ bound, indicating over-parameterization. For this reason, the estimated model order of 3 given by MDL was chosen.

\section{Simulation Results}

In Fig. 3 we can see the estimated attenuation and phase velocity from one simulation using the nonparametric technique. Examining the attenuation estimate, we can see a good fit in the area in which there is a high SNR and poorer, more erratic behavior in the lower SNR regions. In these low SNR regions, a large bias is present, which is the result of trying to estimate the attenuation from just measurement noise on the input and output. This effect shows the importance of uncertainty expressions for the estimates to find its valid frequency region.

The phase velocity figure reveals a similar erratic behavior. Although the bias in this case is a result of the noise-sensitive, phase-unwrapping step, in which the phase unwrapping errors accumulate over the low SNR frequencies, giving a "random walk" behavior. However, this bias is neither an over- nor underestimation, as in the attenuation case, but it fluctuates randomly from simulation to simulation.

In Fig. 4, the attenuation and phase-velocity estimates are shown for the same simulation as in Fig. 3, but using the proposed parametric technique. Compared to the 

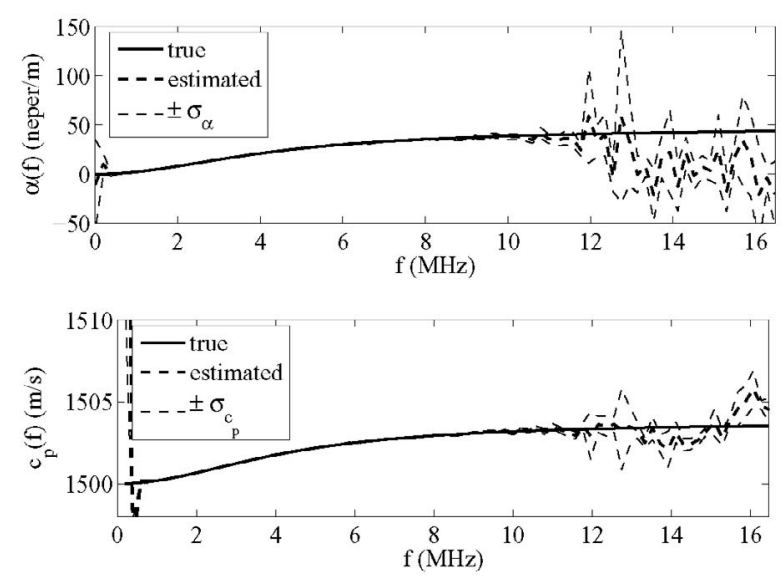

Fig. 3. Nonparametric estimation of the attenuation and phase velocity from one simulation. The bold solid line shows the true parameter, the bold dashed line shows the nonparametric estimation, and the thin dashed line shows the theoretical standard deviation calculated using the expressions in (12).
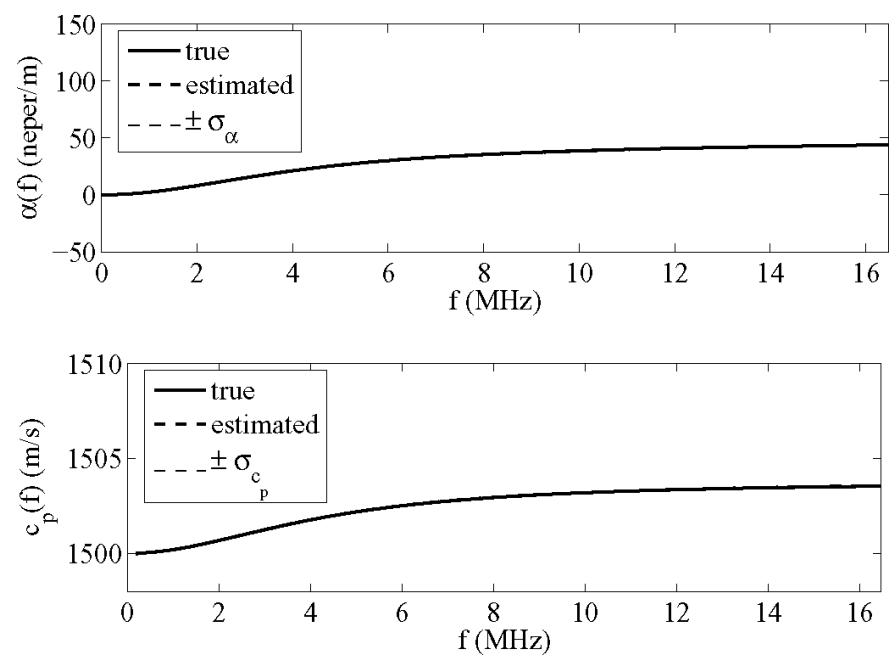

Fig. 4. Parametric estimation of the attenuation and phase velocity from one simulation. The bold solid line is the true parameter, the bold dashed line (coinciding with the bold solid line) is the parametric estimation, and the thin dashed line (coinciding with the bold solid line) is the theoretical standard deviation calculated using (12).

nonparametric estimates, both level of fit and uncertainties are highly improved for both estimates. The improved fit in the low SNR regions is the fruit of weighting the cost function with the inverse of the sample covariance matrix of the noise, in the maximum likelihood estimation of the transfer function in (11), see Appendix A. The overall decrease in standard deviation is the result of using a parameterized model structure compared to a nonparametric estimation procedure. In Fig. 4 it is difficult to see the shape of the standard deviations because the graphs coincide with the plotted quantities at the given scale. A better comparison between both methods standard deviations can be seen in Fig. 5 and Fig. 6 .
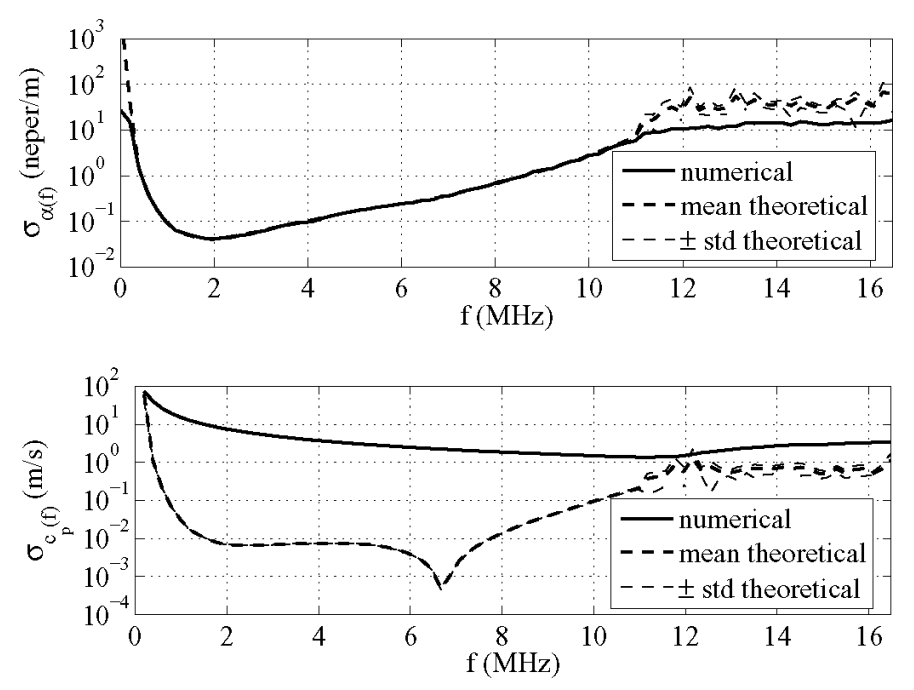

Fig. 5. Nonparametric comparison between the observed numerical and the calculated theoretical standard deviations, for attenuation and phase velocity. The bold solid line is the numerical standard deviation estimated from 500 independent simulations. The bold and thin dashed lines are the mean value and standard deviation of 500 theoretical calculations.

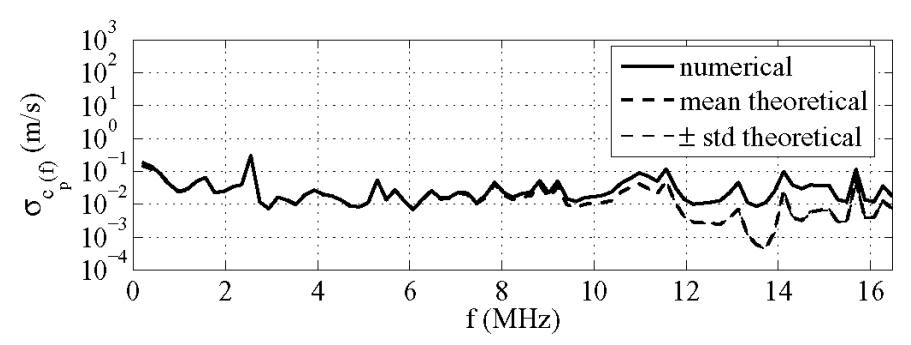

Fig. 6. The same comparison as in Fig. 5, but with a wideband signal (burst of white noise).

\section{Validation of Approximate Uncertainties}

To validate the approximate theoretical expressions of the uncertainties in (12), the observed numerical standard deviation is compared with the mean and standard deviation of theoretical expressions using 500 independent simulations.

In Fig. 5, the numerical and theoretical standard deviations are compared using the nonparametric technique. Examining the attenuation plot, a good fit is obtained in high SNR regions; and an overestimation can be observed in the lower SNR regions. The overestimation is the result of when the statistical linearity fails, and higher order derivatives in (12) must be used.

In the phase-velocity plot, a large deviation can be observed between the theoretical and numerical standard deviations. This is a consequence of the phase-unwrapping step, not taken into account in the theoretical expression. Because the simulated input signal is of bandpass character, the phase-unwrapping errors at the low frequencies (low SNR) accumulate over the entire frequency band, giving high standard deviation even in the high SNR region. To support this explanation, a comparison between the 

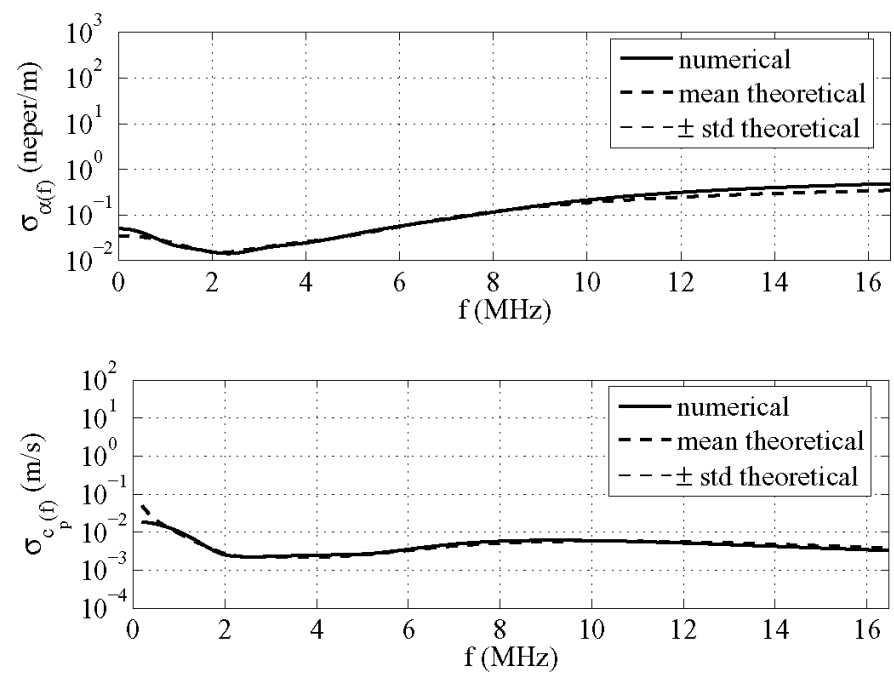

Fig. 7. Parametric comparison between the observed numerical and the calculated theoretical standard deviations for attenuation and phase velocity. The bold solid line is the numerical standard deviation estimated from 500 independent simulations. The bold and thin dashed (coinciding) lines are the mean value and standard deviation of 500 theoretical calculations.

numerical and theoretical standard deviations can be seen in Fig. 7 using simulations with a wideband signal. In this plot only an underestimation can be observed in the highly attenuated (low SNR region) in which the statistical linearity fails.

In the parametric case, a good fit is seen between the approximative theoretical expressions and the observed numerical standard deviation. The good fit is a consequence of an overall low $\sigma_{H(f)}^{2}$ in the parametric case, so the statistical linearity applies over the whole frequency range.

\section{EXPERIMENTS}

To test the method experimentally, experiments were performed in weakly dispersive pulp fiber suspensions. The experiments were carried out using the pulse-echo technique in a custom-designed test cell, shown in Fig. 8. A detailed description and specification of the cell is found in [12].

\section{A. Measurement Setup}

The experimental setup consists of a broadband piezoelectric transducer with a center frequency of $10 \mathrm{MHz}$ (V311), manufactured by Panametrics, Waltham, MA. The transducer is mounted on a buffer rod made of casted plexiglass, and the sample space is located between the buffer rod and a stainless steel reflector, see Fig. 8. A pulser/receiver 5072PR from Panametrics was used to excite the transducer and amplify the received signal. The signal then was digitized using a CompuScope 14100 oscilloscope card, by Gage Applied Technologies Inc., Lachine, QC, Canada, with 14-bit resolution and a sampling rate

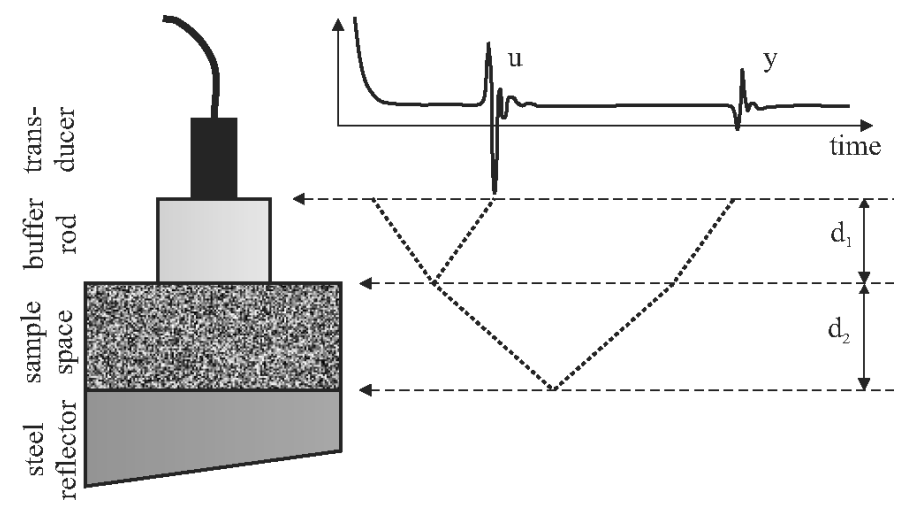

Fig. 8. Lattice diagram of the pulse-echo measurement system used in this study.

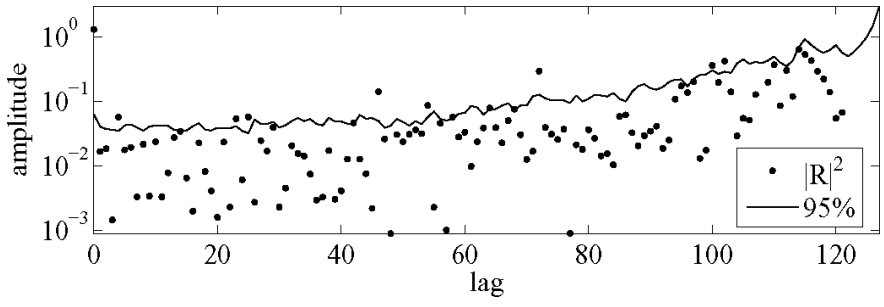

Fig. 9. Whiteness test of the residuals. The dots are the amplitudes of the cross correlation $R$ of the residuals, and the line marks the $95 \%$ confidence level, where $|R|^{2} \sim \chi^{2}(2)$.

of $100 \mathrm{MHz}$. All data were stored in a computer for offline analysis. In the experiment, 100 ultrasonic pulses were captured. A digital thermometer F250, by Automatic Systems Laboratories Ltd., Croydon, England, monitored the temperature both in the suspension under test and in the room. The temperature in the pulp suspension under test was $19.93 \pm 0.03^{\circ} \mathrm{C}$. To accurately determine the distance $d_{2}$ in the cell, pure, distilled water was used as a reference because it has a well-known relationship between speed of sound and temperature [13]. Using the temperature of the calibration fluid and a cross-correlation technique to determine the time-of-flight for an ultrasonic pulse, the distance $d_{2}$ was found to be $0.03010 \pm 0.00004 \mathrm{~m}$. The pulp suspension was carefully poured into the measurement cell, then stirred slowly to remove air bubbles and break up flocs in the suspension.

The pulp samples used in this study were produced from thermomechanical pulp (TMP). The pulp suspension is a $99.5 \%$ water and $0.5 \%$ fiber mixture, with fiber lengths that vary between 1 and $3 \mathrm{~mm}$ and a diameter of 20 $50 \mu \mathrm{m}$. The fiber size distributions were analyzed using a Kajaani Fiberlab instrument, Metso Corporation, Kajaani, Finland.

\section{B. Model Selection and Validation}

In Fig. 9, the result of the whiteness test is presented together with a 95\% confidence level. From this figure, the residual analysis cannot detect any unmodeled dynam- 

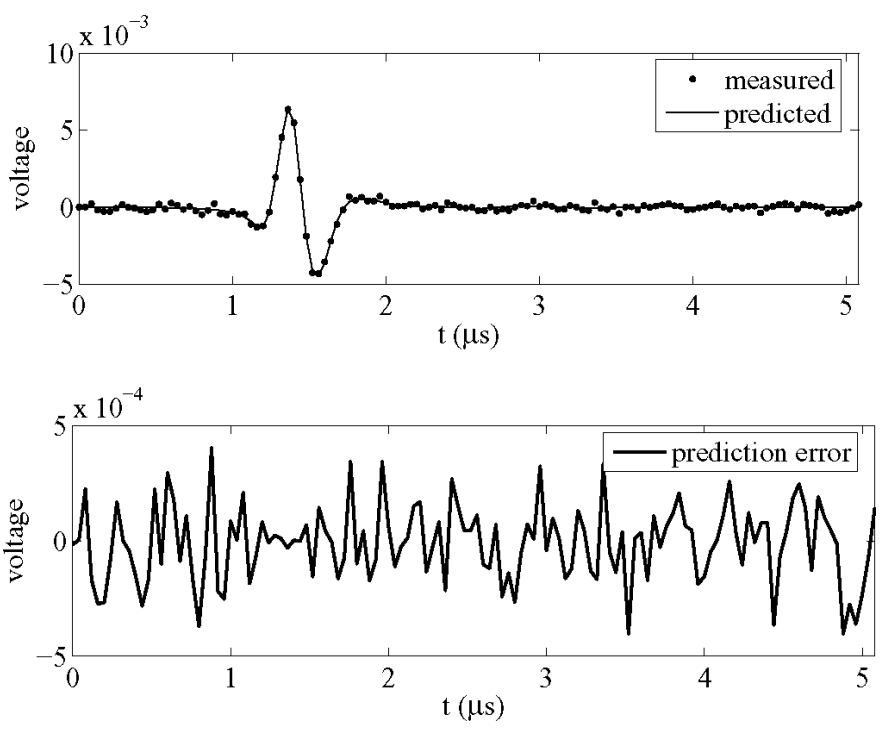

Fig. 10. Comparison between the measured (dots) and predicted (line) second echo. The second echo is predicted using the measured first echo and the estimated parametric model. The lower plot shows the prediction error.

ics. However, the final value of the cost function (146.5) is larger than the expected value (112.6), indicating the presence of nonlinearities [10].

In Fig. 10, the predicted and measured second echo are shown together with the prediction error. The prediction is made using the estimated parametric model and the measured first echo. Examining the graphs, a good fit is obtained by the prediction, and no systematic variation from the pulse shape is visible in the prediction errors (note the difference in scale).

\section{Experimental Results}

In Fig. 11, the estimation results of the attenuation and phase velocity are compared using the nonparametric and parametric techniques. Although the experimental system is quite different from the simulated system, similar behavior of the different estimation techniques is visible. The bias, or underestimation, of the attenuation in the low SNR region is present using the nonparametric technique. Also, the phase-unwrapping problem for the nonparametric technique is visible in the phase-velocity plot, producing a large bias. To support this explanation, the speed of sound is estimated with standard cross correlation and added to the figure. For weakly dispersive fluids, the cross-correlation method estimates an accurate average (over frequency) velocity. The dip in the frequency range $0<f<0.1 \mathrm{MHz}$ for the nonparametric attenuation estimate is the result of both presampling and postsampling processing, i.e., high-pass filtering and detrending to remove unwanted low-frequency disturbance and trends. For this reason, constraints are put on the parametric method to ignore this region in the identification process, explaining its flatness within the region.
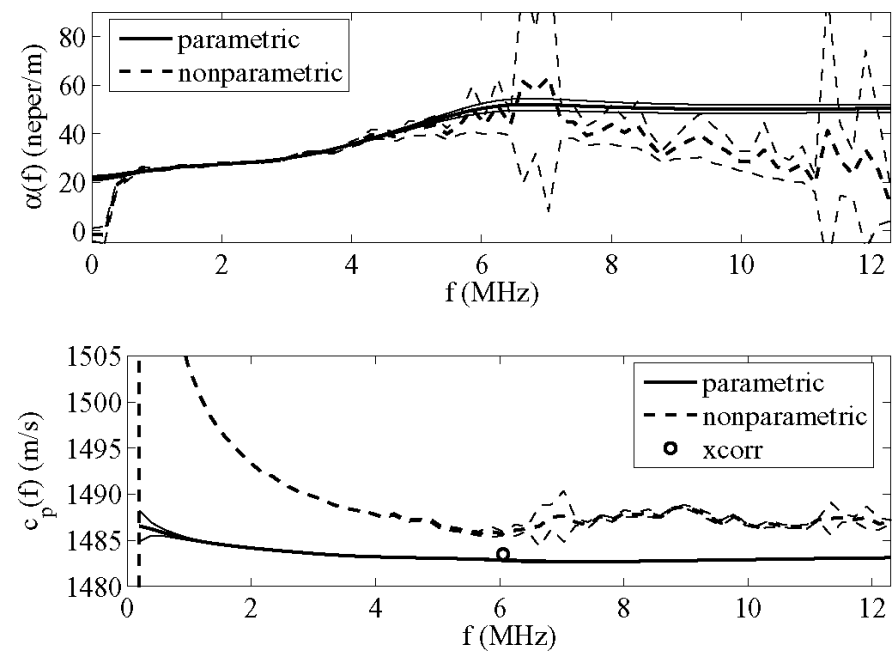

Fig. 11. Comparison between the nonparametric (bold dashed line) and parametric (bold solid line) estimation of attenuation and phase velocity on the experimental data. The thin dashed and solid lines mark the theoretical standard deviation $( \pm \sigma)$. The circle is the speed of sound estimated using standard cross correlation.

\section{Discussion}

The advantages with the proposed method are first, its ability to accurately estimate the frequency-dependent, phase-velocity and attenuation from noisy measurements. Second, its independence of any prior knowledge concerning the measurement setup and physical properties of the medium under investigation, under the condition that the data can be related as in (5). Although its performance is shown under dispersive conditions in simulations, the experimental results are presented from weakly dispersive measurements using only one measurement arrangement. However, as long as the linearity is preserved, more complex dynamic media (including highly dispersive ones) should only increase the model order in the selection and validation step. To further experimentally validate the method, measurements from dispersive media under different experimental arrangements would be of interest. This will be considered in future work. However, due to the generality of the proposed model structure, this should not cause any problems.

When estimating the attenuation and phase velocity in suspensions, it is common to relate or calibrate these estimates to those of pure water to reduce effects from the measurement setup, reflection coefficients, and losses from diffraction. This type of calibration was not applied to the estimation result presented in this paper, as the intention was to compare two different estimation methods. However, if the suspension estimates were to be calibrated to water, the uncertainties of the estimates in water are often negligible in comparison to the suspensions, due to the high SNR difference. The same principles of estimating the transfer function are applied, but with scaled or calibrated input and output signals as $U(\omega)=U_{s}(\omega) / U_{w}(\omega)$ and $Y(\omega)=Y_{s}(\omega) / Y_{w}(\omega)$, where $U_{s}(\omega), Y_{s}(\omega)$ are the measured first and second echoes in the suspension, and 
$U_{w}(\omega), Y_{w}(\omega)$ are the corresponding measurements in distilled water.

However, if the water uncertainties are of the same magnitude as those for the suspension, these uncertainties should be included when calculating the approximate uncertainties in (12). For the nonparametric and parametric methods, the uncertainties can be included using similar approximation as in (22) and (21) in Appendix B.

For the parametric method, high calibration noise has an additional effect of destroying the efficiency of the weighted least squares (WLS) cost-function for the maximum likelihood (ML) method in (15). The WLS cost function is a consequence of identifying the transfer function in the frequency domain. Discrete Fourier transformed measurement noise tends toward normality, by the central limit theorem, resulting in the WLS cost in the frequency domain. However, the calibration or scaling is applied after the DFT, and the quotient changes the normality and thus the efficiency of the cost function. To compensate for the change in the probability densities by changing the costfunction is difficult; hence, it still is recommended to use the WLS due to its favorable properties [11], at the cost of losing some efficiency.

Another approach in this case would be to estimate a separate parametric transfer function for both suspension and water, and do the calibration afterward to preserve normality and efficiency, i.e., $H(\omega)=$ $H_{s}\left(\omega, \boldsymbol{\theta}_{s}\right) / H_{w}\left(\omega, \boldsymbol{\theta}_{v}\right)$, or alternatively using a nonparametric model for water $H(\omega)=H_{s}\left(\omega, \boldsymbol{\theta}_{s}\right) / H_{w}(\omega)$. The drawback compared with prescaling the signals, is an increased complexity of the estimation problem and an increased number of model parameters because prescaling reduces the dynamical content by capturing the differences in the media and ignoring possible linear dynamic effects from the measurement setup.

\section{CONCLUSiOnS}

A comparison between the proposed parametric estimator and the standard nonparametric Fourier analysis technique has been illustrated using both computer simulations and real experiments. The proposed estimator enables accurate estimation of the frequency-dependent attenuation and phase velocity with considerably lower variance compared to standard Fourier analysis methods. Robust phase-velocity estimation is ensured because the phase-unwrapping problem is avoided and replaced by calculation of the phase of an analytical expression. A data reduction with a factor 15 is observed for the present case. This improvement comes at the cost of a model selection and validation step, not necessary for nonparametric methods. Approximate expressions for the uncertainty for both methods were derived, valid for high SNRs or a large number of repeated experiments. The expressions were validated by numerical simulations, showing good accuracy under similar SNR conditions as in the experiment.
APPENDIX A

\section{Maximum Likelihood Cost Function}

The maximum likelihood cost function is expressed as:

$$
V_{M L}(\boldsymbol{\theta})=\sum_{l=0}^{N-1} \frac{\left|E\left(\omega_{l}, \boldsymbol{\theta}\right)\right|^{2}}{\sigma_{E}^{2}\left(\omega_{l}, \boldsymbol{\theta}\right)},
$$

where:

$$
E\left(\omega_{l}, \boldsymbol{\theta}\right)=A\left(\omega_{l}, \boldsymbol{\theta}\right) Y\left(\omega_{l}\right)-B\left(\omega_{l}, \boldsymbol{\theta}\right) e^{-j \omega_{l} \tau} U\left(\omega_{l}\right),
$$

is the equation error and:

$$
\begin{array}{r}
\sigma_{E}^{2}\left(\omega_{l}, \boldsymbol{\theta}\right)=\sigma_{Y}^{2}\left(\omega_{l}\right)\left|A\left(\omega_{l}, \boldsymbol{\theta}\right)\right|^{2}+\sigma_{U}^{2}\left(\omega_{l}\right)\left|B\left(\omega_{l}, \boldsymbol{\theta}\right)\right|^{2} \\
-2 \Re\left\{\sigma_{Y U}^{2}\left(\omega_{l}\right) A\left(\omega_{l}, \boldsymbol{\theta}\right) B^{*}\left(\omega_{l}, \boldsymbol{\theta}\right)\right\},
\end{array}
$$

its uncertainty. Estimations for $\sigma_{U}^{2}\left(\omega_{l}\right), \sigma_{Y}^{2}\left(\omega_{l}\right)$ and $\sigma_{Y U}^{2}\left(\omega_{l}\right)$ are found in Appendix B.

\section{APPENDIX B}

\section{Calculation of Approximate Uncertainties}

The partial derivatives in (7) w.r.t $H(\omega)$ are given by (omitting $\omega$ as an argument in the expressions):

$$
\begin{aligned}
\frac{\partial \alpha(H)}{\partial H} & =-\frac{1}{2 x H} \\
\frac{\partial c_{p}(H)}{\partial H} & =-\frac{x j \omega}{2 \arg \{H\}^{2} H} .
\end{aligned}
$$

Here a complex derivative of a real scalar function $f$ w.r.t a complex parameter $z$ is defined as:

$$
\frac{\partial f(z)}{\partial z}=\frac{1}{2}\left[\frac{\partial f(z)}{\partial a}-j \frac{\partial f(z)}{\partial b}\right],
$$

where $z=a+j b$ with $a$ and $b$ being the real and imaginary parts of $z$, respectively.

The estimated uncertainty for the input and output measurement noise is estimated using the sample variance. Given a set of $M$ independent noisy but identically excited experiments, the input and output noise variances are estimated as:

$$
\begin{aligned}
\hat{\sigma}_{U}^{2} & =\frac{1}{M-1} \sum_{m=1}^{M}\left|U_{m}-\bar{U}\right|^{2}, \\
\hat{\sigma}_{Y}^{2} & =\frac{1}{M-1} \sum_{m=1}^{M}\left|Y_{m}-\bar{Y}\right|^{2}, \\
\hat{\sigma}_{Y U}^{2} & =\frac{1}{M-1} \sum_{m=1}^{M}\left[Y_{m}-\bar{Y}\right]\left[U_{m}^{*}-\bar{U}^{*}\right],
\end{aligned}
$$

where $U_{m}$ and $Y_{m}$ represent the discrete Fourier transforms of the p:th input and output signal, and $\bar{U}, \bar{Y}$ denotes the sample mean (over $M$ experiments). 
The estimated uncertainty for the nonparametric transfer function is estimated using a first order Taylor series expansion of the transfer function in (8), including measurement noise as:

$$
\begin{aligned}
H & =\frac{Y}{U}=\frac{Y_{0}+N_{Y}}{U_{0}+N_{U}}=\frac{Y_{0}\left[1+N_{Y} / Y_{0}\right]}{U_{0}\left[1+N_{U} / U_{0}\right]} \\
& \approx \frac{Y_{0}}{U_{0}}\left[1+N_{Y} / Y_{0}-N_{U} / U_{0}\right],
\end{aligned}
$$

where $U_{0}, Y_{0}$ represents the noise-free signals, and $N_{U}, N_{Y}$ represent the measurement noise. An approximative uncertainty for $H$ can be expressed using (22) as:

$$
\begin{aligned}
\sigma_{H}^{2} & =E\left\{|H-E\{H\}|^{2}\right\} \\
& \approx\left|\frac{Y_{0}}{U_{0}}\right|^{2}\left[\frac{\sigma_{Y}^{2}}{\left|Y_{0}\right|^{2}}+\frac{\sigma_{U}^{2}}{\left|U_{0}\right|^{2}}-2 \Re\left\{\frac{\sigma_{Y U}^{2}}{Y_{0} U_{0}^{*}}\right\}\right],
\end{aligned}
$$

where $E\{\cdot\}$ denotes expectation. The estimated uncertainty is obtained by dividing (23) with $M$ and replacing $U_{0}, Y_{0}$ with $\bar{Y}, \bar{U}$ and using the estimated measurement uncertainties (21).

The parametric transfer function uncertainty is estimated from (11) using a first order Taylor approximation as:

$$
\sigma_{H}^{2} \approx \frac{\partial H}{\partial \boldsymbol{\theta}} C_{\theta}\left[\frac{\partial H}{\partial \boldsymbol{\theta}}\right]^{*}
$$

where * $^{*}$ denotes Hermitian transpose. An approximate expression for the covariance matrix of $\boldsymbol{\theta}$ can be obtained from an approximation of the Hessian (Fisher information matrix) of the cost-function [in (15)] as:

$$
C_{\theta} \approx \frac{1}{2} \Re\left\{\left[\frac{\partial}{\partial \boldsymbol{\theta}}\left(\frac{E(\boldsymbol{\theta})}{\sigma_{E}(\boldsymbol{\theta})}\right)\right]^{*}\left[\frac{\partial}{\partial \boldsymbol{\theta}}\left(\frac{E(\boldsymbol{\theta})}{\sigma_{E}(\boldsymbol{\theta})}\right)\right]\right\}^{-1} .
$$

The expressions for $E(\boldsymbol{\theta})$ and $\sigma_{E}(\boldsymbol{\theta})$ can be found in Appendix A.

\section{ACKNowledgments}

Generous grants from the Swedish Energy Agency and the Kempe Foundation are gratefully acknowledged.

\section{REFERENCES}

[1] D. R. Brillinger, Time Series: Data Analysis and Theory. (Holden-Day series in time series analysis.) San Francisco: Holden-Day, 1981.

[2] L. Ljung, "On the estimation of transfer functions," Automatica, vol. 21, no. 6, pp. 677-696, 1985.

[3] J. M. Tribolet, "A new phase unwrapping algorithm," IEEE Trans. Acoust. Speech Signal Processing, vol. ASSP-25, pp. 170177, Apr. 1977.

[4] M. O'Donnell, E. T. Jayes, and J. G. Miller, "Kramers-Kronig relationship between ultrasonic attenuation and phase velocity," J. Acoust. Soc. Amer., vol. 69, no. 3, pp. 696-701, 1981.
[5] P. He, "Experimental verification of models for determining dispersion from attenuation," IEEE Trans. Ultrason., Ferroelect., Freq. Contr., vol. 46, no. 3, pp. 706-714, 1999.

[6] J. Niemi, Y. Aitomäki, and T. Löfqvist, "Ultrasonic measurements and modelling of attenuation and phase velocity in pulp suspensions," in Proc. IEEE Ultrason. Symp., 2005, pp. 775779 .

[7] A. N. Kalashnikov and R. E. Challis, "Errors and uncertainties in the measurement of ultrasonic wave attenuation and phase velocity," IEEE Trans. Ultrason., Ferroelect., Freq. Contr., vol. 52 , pp. $1754-1768$, Oct. 2005.

[8] H. Jeffreys and B. Swirles, Methods of Mathematical Physics. 3rd ed. Cambridge: Cambridge Univ. Press, 1956.

[9] J. Martinsson and J. E. Carlson, "Parametric modeling of wave propagation in gas mixtures-A system identification approach," in Proc. IEEE Ultrason. Symp., 2005, pp. 2288-2292.

[10] R. Pintelton and J. Schoukens, System Identification: A Frequency Domain Approach. New York: IEEE Press, 2001.

[11] S. M. Kay, Fundamentals of Statistical Signal Processing: Estimation Theory. vol. 1, Englewood Cliffs, NJ: Prentice-Hall, 1993.

[12] T. Löfqvist, "Ultrasonic wave attenuation and phase velocity in a paper-fibre suspension," in Proc. IEEE Ultrason. Symp., 1997, pp. 841-844.

[13] N. Bilaniuk and G. S. K. Wong, "Speed of sound in pure water as a function of temperature," J. Acoust. Soc. Amer., vol. 93, no. 3, pp. 1609-1612, 1993.

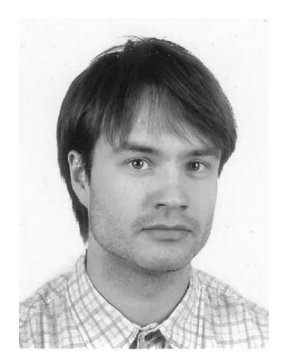

Jesper Martinsson was born in Gällivare, Sweden, in 1978. He received his M.Sc. degree in computer science and engineering in 2004, and a Licentiate degree in electrical engineering in 2006, from Luleå University of Technology, Luleå, Sweden. He is currently pursuing a Ph.D degree in electrical engineering at Luleå University of Technology.

His research interests include ultrasonics, signal processing, and measurement technology.

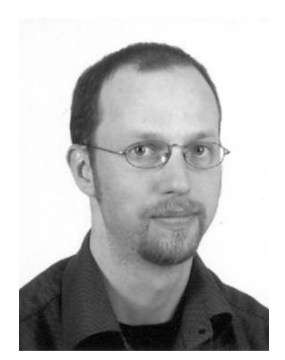

Johan E. Carlson was born 1973 in Umeå, Sweden. He received his M.Sc. degree in computer science and engineering (1998) and Ph.D. degree in electrical engineering (2002), at Luleå University of Technology, Luleå, Sweden.

In 2006 he was appointed Associate Professor and received the young researcher award from the Royal Skyttean Society. He currently holds a position as senior lecturer at the Department of Computer Science and Electrical Engineering at Luleå University of Technology. His research interests include: ultrasonic measurement technology, signal processing, and multivariate data analysis.

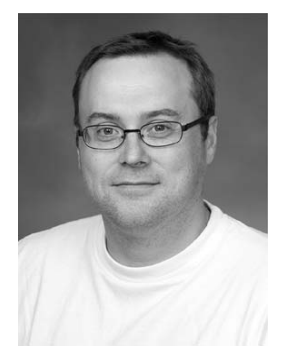

Jan Niemi was born in Kiruna, Sweden, in 1967. He received the M.Sc. degree in electrical engineering from Luleå University of Technology, Luleå, Sweden, in 2004. Since 2004 he has been a Ph.D. student at the Department of Computer Science and Electrical Engineering at Luleå University of Technology.

His research interest is on developing a sensor for the paper pulp industry using a combination of ultrasound and optics. 